\title{
Modified Block Pulse Functions for Numerical Solution of Stochastic Volterra Integral Equations
}

\author{
K. Maleknejad, M. Khodabin, and F. Hosseini Shekarabi \\ Department of Mathematics, Islamic Azad University, Karaj Branch, Karaj, Iran \\ Correspondence should be addressed to K. Maleknejad; maleknejad@iust.ac.ir \\ Received 13 December 2013; Accepted 22 January 2014; Published 13 March 2014 \\ Academic Editor: Kai Diethelm
}

Copyright (c) 2014 K. Maleknejad et al. This is an open access article distributed under the Creative Commons Attribution License, which permits unrestricted use, distribution, and reproduction in any medium, provided the original work is properly cited.

We present a new technique for solving numerically stochastic Volterra integral equation based on modified block pulse functions. It declares that the rate of convergence of the presented method is faster than the method based on block pulse functions. Efficiency of this method and good degree of accuracy are confirmed by a numerical example.

\section{Introduction}

The numerical study and simulation of stochastic Volterra integral equations (SVIEs) have been an active field of research for the past years [1-7]. Most SVIEs do not have analytic solutions and hence it is of great importance to provide numerical schemes. Numerical schemes to stochastic differential equations (SDEs) have been well developed [812]. However, there are still few papers discussing the numerical solutions for stochastic Volterra integral equations.

Study in economics, sociology, and various biological and medical models leads to the stochastic Volterra integral equations. These systems are dependent on a noise source, on a Gaussian white noise, so that modeling such phenomena naturally requires the use of various stochastic Volterra integral equations.

In this paper, we consider the linear stochastic Volterra integral equation:

$$
\begin{aligned}
u(t)= & u_{0}(t)+\int_{0}^{t} k_{1}(s, t) u(s) d s \\
& +\int_{0}^{t} k_{2}(s, t) u(s) d B(s) \quad t \in[0, T],
\end{aligned}
$$

where $u(t), u_{0}(t), k_{1}(s, t)$, and $k_{2}(s, t)$, for $s, t \in[0, T)$, are the stochastic processes defined on the same probability space $(\Omega, \mathscr{F}, P)$ with a filtration $\left\{\mathscr{F}_{t}, t \geq 0\right\}$ that is increasing and right continuous and $\mathscr{F}_{0}$ contains all $P$-null sets. $u(t)$ is unknown random function and $B(t)$ is a standard Brownian motion defined on the probability space and $\int_{0}^{t} k_{2}(s, t) u(s) d B(s)$ is the Itô integral. Numerous papers have been focusing on existence solution of (1) [13-15].

The paper [3] solves stochastic Volterra integral equations by block pulse functions (BPFs) and [4] applies this method for solving $m$-dimensional stochastic Itô Volterra integral equations. However, BPFs are very common in use; it seems that their convergence is weak. Maleknejad and Rahimi apply in [16] $\varepsilon$ Modified Block Pulse Functions ( $\varepsilon$ MBPFs) to solve Volterra integral equation of the first kind numerically. Here, we use this method for solving SVIEs.

This paper is organized as follows. In the rest of this section we describe some general concepts concerning the block pulse functions and epsilon modified block pulse functions and some concepts related to stochastic and Itô integral. Section 2 is devoted to stochastic integration operational matrix. In Section 3, the method is employed to solve stochastic integral equations. Section 4 discusses error analysis of this method. Section 5 gives numerical example. Finally, Section 6 provides the conclusion of this work.

1.1. Block Pulse Functions. BPFs have been variously studied [16-18] and applied for solving different problems. The goal of this section is to recall notations and definition of the BPFs that are used in the next sections. 
The block pulse functions are defined on the time interval $[0, T)$ by

$$
\psi_{i}(t)= \begin{cases}1 & (i-1) \frac{T}{m} \leq t<i \frac{T}{m} \\ 0 & \text { elsewhere }\end{cases}
$$

where $i=1, \ldots, m$ and for convenience we put $h=T / m$.

The block pulse functions on $[0, T)$ have the following properties:

(1) disjointness: for $i, j=1, \ldots, m$

$$
\psi_{i}(t) \psi_{j}(t)=\delta_{i j} \psi_{i}(t)
$$

where $\delta_{i j}$ is Kronecker delta;

(2) orthogonality:

$$
\int_{0}^{T} \psi_{i}(t) \psi_{j}(t) d t=\delta_{i j} h
$$

(3) completeness: for every $f \in L^{2}([0, T))$ when $m$ approaches infinity, Parseval's identity holds:

$$
\int_{0}^{T} f^{2}(t) d t=\lim _{m \rightarrow \infty} \sum_{i=1}^{m}\left(f_{i}\right)^{2}\left\|\psi_{i}(t)\right\|^{2},
$$

where

$$
f_{i}=\frac{1}{h} \int_{0}^{T} f(t) \psi_{i}(t) d t
$$

Also the Fourier coefficients $f_{i}$ and the block pulse functions depend on $m$. The set of block pulse functions may be written as a vector $\Psi(t)$ of dimension $m$ :

$$
\Psi(t)=\left[\psi_{1}(t), \ldots, \psi_{m}(t)\right]^{T} \quad t \in[0, T) .
$$

From the above representation and disjointness property, it follows that

$$
\begin{gathered}
\Psi(t) \Psi^{T}(t)=\left(\begin{array}{ccccc}
\psi_{1}(t) & 0 & 0 & \cdots & 0 \\
0 & \psi_{2}(t) & 0 & \cdots & 0 \\
\vdots & \vdots & \vdots & \ddots & \vdots \\
0 & 0 & 0 & \cdots & \psi_{m}(t)
\end{array}\right)_{m \times m} \\
\Psi^{T}(t) \Psi(t)=1, \\
\Psi(t) \Psi^{T}(t) F=D_{F} \Psi(t),
\end{gathered}
$$

where $F$ is an $m$-dimensional vector and $D_{F}=\operatorname{diag}(F)$. Let $G$ be an $m \times m$ matrix so that

$$
\Psi^{T}(t) G \Psi(t)=\widehat{G}^{T} \Psi(t),
$$

where $\widehat{G}$ is a vector with elements equal to the diagonal entries of $G$.
The expansion of a function $f(t)$ over $[0, T)$ with respect to $\psi_{i}(t), i=1, \ldots, m$, is given by

$$
f(t) \simeq \sum_{i=1}^{m} f_{i} \psi_{i}(t)=F^{T} \Psi(t)=\Psi^{T}(t) F,
$$

where $F=\left[f_{1}, \ldots, f_{m}\right]^{T}$ and $f_{i}$ is defined by (6).

Let $k(s, t) \in L^{2}\left(\left[0, T_{1}\right) \times\left[0, T_{2}\right)\right)$. It is expanded with respect to BPFs as

$$
k(s, t) \simeq \Psi^{T}(s) K \Lambda(t),
$$

where $\Psi(s)$ and $\Lambda(t)$ are $m_{1}$ - and $m_{2}$-dimensional BPFs vectors, respectively, and $K$ is the $m_{1} \times m_{2}$ block pulse coefficient matrix with the below $k_{i j}, i=1, \ldots, m_{1}, j=1, \ldots, m_{2}$ :

$$
k_{i j}=\frac{m_{1} m_{2}}{T_{1} T_{2}} \int_{0}^{T_{1}} \int_{0}^{T_{2}} k(s, t) \psi_{i}(s) \lambda_{j}(t) d s d t .
$$

For convenience, we put $m_{1}=m_{2}=m$.

Now, integration operational matrix is considered and computed:

$$
\int_{0}^{t} \psi_{i}(s) d s= \begin{cases}0 & 0 \leq t \leq(i-1) h, \\ t-(i-1) h & (i-1) h \leq t \leq i h, \\ h & i h \leq t<1 .\end{cases}
$$

Since $t-i h$ is equal to $h / 2$ at midpoint of $[i h,(i+1) h)$, we can approximate $t-(i-1) h$, for $(i-1) h \leq t<i h$ by $h / 2$. Therefore

$$
\int_{0}^{t} \psi_{i}(s) d s \simeq\left(0, \ldots, 0, \frac{h}{2}, h, \ldots, h\right) \Psi(t),
$$

where the $i$ th component is $h / 2$. As a result

$$
\int_{0}^{t} \Psi(s) d s \simeq Q \Psi(t)
$$

where $Q$ is operational matrix of integration that is given by

$$
Q=\frac{h}{2}\left(\begin{array}{ccccc}
1 & 2 & 2 & \cdots & 2 \\
0 & 1 & 2 & \cdots & 2 \\
0 & 0 & 1 & \cdots & 2 \\
\vdots & \vdots & \vdots & \ddots & \vdots \\
0 & 0 & 0 & \cdots & 1
\end{array}\right)
$$

So,

$$
\int_{0}^{t} f(s) d s \simeq \int_{0}^{t} F^{T} \Psi(s) d s \simeq F^{T} Q \Psi(t) .
$$

1.2. Epsilon Modified Block Pulse Functions (EMBPFs). A set of epsilon modified block pulse functions $\theta_{i}(t), i=$ $0,1, \ldots, m$, on the interval $[0, T)$ are defined as

$$
\theta_{0}(t)= \begin{cases}1 & t \in\left[0, \frac{T}{m}-\varepsilon\right)=I_{0} \\ 0 & \text { otherwise }\end{cases}
$$

$$
\theta_{i}(t)= \begin{cases}1 & t \in\left[\frac{i T}{m}-\varepsilon, \frac{(i+1) T}{m}-\varepsilon\right)=I_{i} \\ 0 & \text { otherwise }\end{cases}
$$


for $i=1, . ., m-1$, and

$$
\theta_{m}(t)= \begin{cases}1 & t \in[T-\varepsilon, T)=I_{m} \\ 0 & \text { otherwise }\end{cases}
$$

Similar to BPFs, the most important properties of EMBPFs are

(1) disjointness:

$$
\theta_{i}(t) \theta_{j}(t)= \begin{cases}\theta_{i}(t) & i=j, \\ 0 & i \neq j,\end{cases}
$$

where $i, j=0, \ldots, m$;

(2) orthogonality: if we put $h=T / m$,

$$
\int_{0}^{T} \theta_{i}(t) \theta_{j}(t) d t=h \delta_{i j}, \quad i, j=1, \ldots, m-1 ;
$$

(3) completeness:

$$
\int_{0}^{T} f^{2}(t) d t=\sum_{i=0}^{\infty} f_{i}^{2}\left\|\theta_{i}(t)\right\|^{2}
$$

where

$$
f_{i}=\frac{1}{\Delta\left(I_{i}\right)} \int_{0}^{T} f(t) \theta_{i}(t) d t
$$

and $\Delta\left(I_{i}\right)$ is length of interval $I_{i}$.

With defining $\Theta_{m+1}(t)=\left[\theta_{0}(t), \ldots, \theta_{m}(t)\right]^{T}$, we have

$$
\begin{aligned}
\Theta_{m+1}(t) \Theta_{m+1}^{T}(t) & \\
= & \left(\begin{array}{ccccc}
\theta_{0}(t) & 0 & 0 & \cdots & 0 \\
0 & \theta_{1}(t) & 0 & \cdots & 0 \\
\vdots & \vdots & \vdots & \ddots & \vdots \\
0 & 0 & 0 & \cdots & \theta_{m}(t)
\end{array}\right)_{m+1 \times m+1} \\
\Theta_{m+1}^{T}(t) \Theta_{m+1}(t)=1, & \\
& \Theta_{m+1}(t) \Theta_{m+1}^{T}(t) F=D_{F} \Theta_{m+1}(t), \\
\Theta_{m+1}^{T}(t) G \Theta_{m+1}(t) & =\widehat{G}^{T} \Theta_{m+1}(t) .
\end{aligned}
$$

Similar to BPFs,

$$
\int_{0}^{t} \Theta_{m+1}(s) d s \simeq P \Theta_{m+1}(t),
$$

where the operational matrix $P$ of EMBPFs is given by

$$
P=\left(\begin{array}{ccccc}
\frac{h-\varepsilon}{2} & h-\varepsilon & \cdots & h-\varepsilon & h-\varepsilon \\
0 & \frac{h}{2} & h & \cdots & h \\
0 & 0 & \frac{h}{2} & \cdots & h \\
\vdots & \vdots & \vdots & \ddots & \vdots \\
0 & 0 & 0 & \cdots & \frac{\varepsilon}{2}
\end{array}\right)_{m+1 \times m+1}
$$

and we have the following approximation:

$$
\int_{0}^{t} f(s) d s \simeq \int_{0}^{t} F^{T} \Theta_{m+1}(s) d s \simeq F^{T} P \Theta_{m+1}(s) .
$$

\subsection{Stochastic Concepts of Itô Integral}

Definition 1 (Brownian motion process). A real-valued stochastic process $\{B(t), t \geq 0\}$ is called Brownian motion, if it satisfies the following properties:

(i) independence of increments: $B(t)-B(s), t>s$, is independent of the past, that is, of $B(u), 0 \leq u \leq s$, or of $\mathscr{F}_{s}$, the $\sigma$-field generated by $B(u), u \leq s$;

(ii) normal increments: $B(t)-B(s)$ has normal distribution with mean 0 and variance $t-s$;

(iii) continuity of paths: $B(t), t \geq 0$, are continuous functions of $t$.

Definition 2. Let $\{N(t)\}_{t \geq 0}$ be an increasing family of $\sigma$-algebras of subsets of $\Omega$. A process $g(t, \omega)$ from $[0, \infty) \times \Omega$ to $R^{n}$ is called $N(t)$-adapted if for each $t \geq 0$ the function $\omega \mapsto g(t, \omega)$ is $N(t)$-measurable [19].

Definition 3 (see [19]). Let $v=v(S, T)$ be the class of functions $f(t, \omega):[0, \infty) \times \Omega \rightarrow R$ such that

(i) $(t, \omega) \mapsto f(t, \omega)$ is $B \times \mathscr{F}$-measurable, where $B$ denotes the Borel $\sigma$-algebra on $[0, \infty)$ and $\mathscr{F}$ is the $\sigma$-algebra on $\Omega$;

(ii) $f(t, \omega)$ is $\mathscr{F}_{t}$-adapted, where $\mathscr{F}_{t}$ is the $\sigma$-algebra generated by the random variables $B(s), s \leq t$;

(iii) $E\left[\int_{S}^{T} f^{2}(t, \omega) d t\right]<\infty$.

Definition 4 (the Itô integral, [19]). Let $f \in \nu(S, T)$; then the Itô integral of $f$ (from $S$ to $T$ ) is defined by

$$
\int_{S}^{T} f(t, \omega) d B(t)(\omega)=\lim _{n \rightarrow \infty} \int_{S}^{T} \phi_{n}(t, \omega) d B(t)(\omega),
$$

(limit in $\left.L^{2}(P)\right)$, 
where $\phi_{n}$ is a sequence of elementary functions such that

$$
E\left[\int_{S}^{T}\left(f(t, \omega)-\phi_{n}(t, \omega)\right)^{2} d t\right] \longrightarrow 0, \quad \text { as } n \longrightarrow \infty .
$$

Theorem 5 (the Itô isometry). Let $f \in \nu(S, T)$; then

$$
\begin{gathered}
E\left[\left(\int_{S}^{T} f(t, \omega) d B(t)(w)\right)^{2}\right] \\
=E\left[\int_{S}^{T} f^{2}(t, \omega) d(t)\right] .
\end{gathered}
$$

Proof. See [19].

Definition 6 (1-dimensional Itô processes, [19]). Let $B(t)$ be 1dimensional Brownian motion on $(\Omega, \mathscr{F}, P)$. A 1-dimensional Itô process (stochastic integral) is a stochastic process $X(t)$ on $(\Omega, \mathscr{F}, P)$ of the form

$$
X(t)=X(0)+\int_{0}^{t} u(s, \omega) d s+\int_{0}^{t} v(s, \omega) d B(s),
$$

or

$$
d X(t)=u d t+v d B(t)
$$

where

$$
\begin{aligned}
& P\left[\int_{0}^{t} v^{2}(s, \omega) d s<\infty, \forall t \geq 0\right]=1, \\
& P\left[\int_{0}^{t}|u(s, \omega)| d s<\infty, \forall t \geq 0\right]=1 .
\end{aligned}
$$

Theorem 7 (the 1-dimensional Itô formula). Let $X(t)$ be an Itô process given by $(1)$ and $g(t, x) \in C^{2}([0, \infty) \times R)$; then

$$
Y(t)=g(t, X(t))
$$

is again an Itô process, and

$$
\begin{aligned}
d Y(t)= & \frac{\partial g}{\partial t}(t, X(t)) d t+\frac{\partial g}{\partial x}(t, X(t)) d X(t) \\
& +\frac{1}{2} \frac{\partial^{2} g}{\partial x^{2}}(t, X(t))(d X(t))^{2},
\end{aligned}
$$

where $(d X(t))^{2}=(d X(t))(d X(t))$ is computed according to the rules

$$
\begin{gathered}
d t \cdot d t=d t \cdot d B(t)=d B(t) \cdot d t=0, \\
d B(t) \cdot d B(t)=d t .
\end{gathered}
$$

Proof. See [19].

Moreover, $\|\cdot\|$ is notation of

$$
\|f(t)\|^{2}=\int_{0}^{1}|f(t)|^{2} d t .
$$

Lemma 8 (the Gronwall inequality). Let $\alpha, \beta \in\left[t_{0}, T\right] \rightarrow R$ be integral with

$$
0 \leq \alpha(t) \leq \beta(t)+L \int_{t_{0}}^{t} \alpha(s) d s
$$

for $t \in\left[t_{0}, T\right]$, where $L>0$. Then

$$
\alpha(t) \leq \beta(t)+L \int_{t_{0}}^{t} e^{L(t-s)} \beta(s) d s, \quad t \in\left[t_{0}, T\right] .
$$

For more details see $[19,20]$.

\section{Stochastic Integral Operational Matrix for EMBPFs}

In this section stochastic integral operational matrix for EMBPFs is considered. For finding vector form of $\int_{0}^{t} \theta_{i}(s) d B(s)$, with EMBPFs, the Itô integral of each single EMBPF $\theta_{i}(t)$ can be computed as follows. It is clear that the integrals are stochastic and nondeterministic:

$$
\begin{gathered}
\int_{0}^{t} \theta_{0}(s) d B(s)= \begin{cases}B(t)-B(0) & 0 \leq t<h-\varepsilon, \\
B(h-\varepsilon)-B(0) & h-\varepsilon \leq t<T .\end{cases} \\
\int_{0}^{t} \theta_{i}(s) d B(s) \\
= \begin{cases}0 & 0 \leq t<i h-\varepsilon, \\
B(t)-B(i h-\varepsilon) & i h-\varepsilon \leq t<(i+1) h-\varepsilon, \\
B((i+1) h-\varepsilon)-B(i h-\varepsilon) & (i+1) h-\varepsilon \leq t<T,\end{cases}
\end{gathered}
$$

for $i=1, \ldots, m$, and

$$
\begin{aligned}
\int_{0}^{t} \theta_{m}(s) d B(s) & \\
= & \begin{cases}0 & 0 \leq t<T-\varepsilon, \\
B(t)-B(T-\varepsilon) & T-\varepsilon \leq t<T .\end{cases}
\end{aligned}
$$

We approximate

(1) $B(t)-B(i h-\varepsilon)$, by $B((i+0.5) h-\varepsilon)-B(i h-\varepsilon)$, at midpoint of $[i h-\varepsilon,(i+1) h-\varepsilon)$;

(2) $B(t)-B(0)$ by $B((h-\varepsilon) / 2)$ in $\theta_{0}(t)$ at midpoint of $[0, h-$ $\varepsilon)$;

(3) $B(t)-B(T-\varepsilon)$ by $B(T-(\varepsilon / 2))-B(T-\varepsilon)$ in $\theta_{m}(t)$, at midpoint of $[T-\varepsilon, T)$. 
As a result, vector form of $\int_{0}^{t} \theta_{i}(s) d B(s)$, with EMBPFs, is given by

$$
\begin{gathered}
\int_{0}^{t} \theta_{0}(s) d B(s) \\
\simeq\left(B\left(\frac{h-\varepsilon}{2}\right), B(h-\varepsilon), \ldots, B(h-\varepsilon)\right) \Theta(t), \\
\int_{0}^{t} \theta_{i}(s) d B(s) \\
\simeq(0,0, \ldots, 0, B((i+0.5) h-\varepsilon)-B(i h-\varepsilon), \\
B((i+1) h-\varepsilon)-B(i h-\varepsilon), \ldots, \\
B((i+1) h-\varepsilon)-B(i h-\varepsilon)) \Theta(t),
\end{gathered}
$$

Therefore

$$
\int_{0}^{t} \Theta(s) d B(s) \simeq P_{S} \Theta(t)
$$

where stochastic operational matrix of integration is given by

$$
\simeq\left(0,0, \ldots, B\left(T-\frac{\varepsilon}{2}\right)-B(T-\varepsilon)\right) \Theta(t) .
$$

$$
P_{S}=\left(\begin{array}{ccccc}
B\left(\frac{h-\varepsilon}{2}\right) & B(h-\varepsilon) & B(h-\varepsilon) & \cdots & B(h) \\
0 & B\left(\frac{3 h}{2}-\varepsilon\right)-B(h-\varepsilon) & B(2 h-\varepsilon)-B(h-\varepsilon) & \ldots & B(2 h-\varepsilon)-B(h-\varepsilon) \\
0 & 0 & B\left(\frac{5 h}{2}-\varepsilon\right)-B(2 h-\varepsilon) & \cdots & B(3 h-\varepsilon)-B(2 h-\varepsilon) \\
\vdots & \vdots & \vdots & \ddots & \vdots \\
0 & 0 & 0 & \cdots & B\left(\frac{(2 m-1) h}{2}-\varepsilon\right)-B((m-1) h-\varepsilon) \\
0 & 0 & 0 & \cdots & B\left(T-\frac{\varepsilon}{2}\right)-B(T-\varepsilon)
\end{array}\right)
$$




$$
\begin{aligned}
& B=\left(\begin{array}{ccccc}
k_{00}^{1}\left(\frac{h-\varepsilon}{2}\right) & k_{01}^{1}(h-\varepsilon) & k_{02}^{1}(h-\varepsilon) & \cdots & k_{0 m}^{1}(h-\varepsilon) \\
0 & k_{11}^{1}\left(\frac{h}{2}\right) & 2 k_{12}^{1} h & \cdots & 2 k_{1 m}^{1} h \\
0 & 0 & k_{33}^{1} \frac{h}{2} & \cdots & 2 k_{3 m}^{1} h \\
\vdots & \vdots & \vdots & \ddots & \vdots \\
0 & 0 & 0 & \cdots & k_{m m}^{1} \frac{\varepsilon}{2}
\end{array}\right)_{m+1 \times m+1}
\end{aligned}
$$

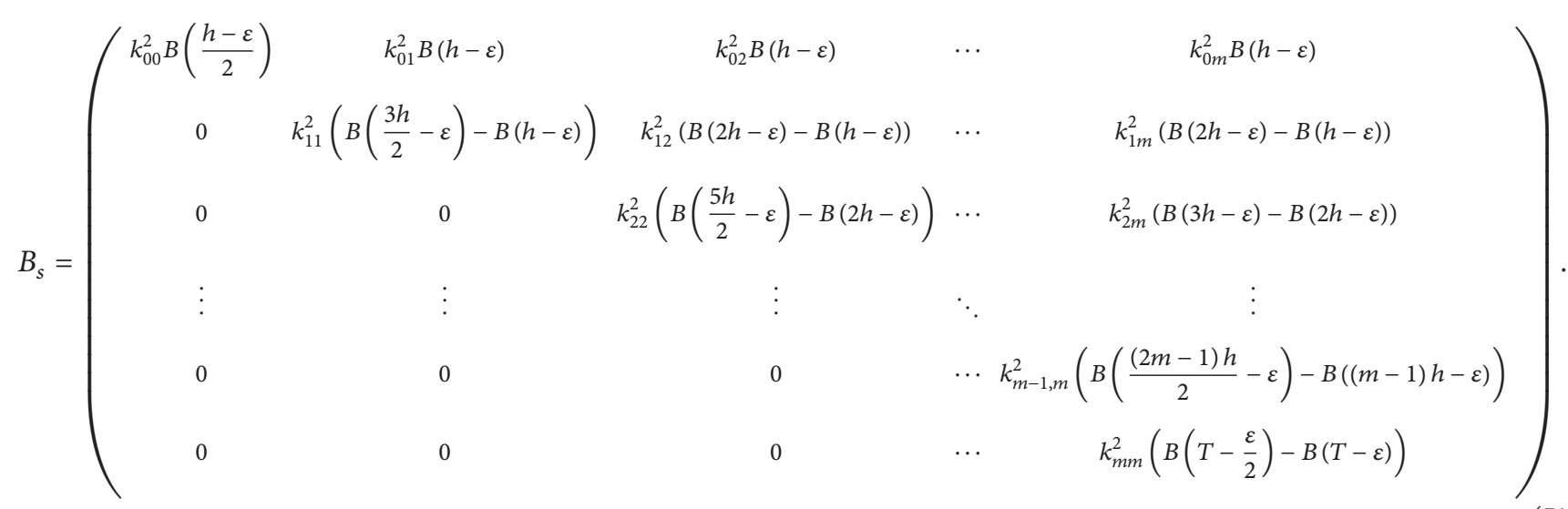

Then

$$
U^{T}\left(I-B-B_{s}\right) \simeq U_{0} .
$$

With replacing $\simeq$ by $=$, we have a linear system of equations.

Now if $\varepsilon_{j}=j h / k, j=0,1, \ldots, k-1$, there will be $k$ numerical answers $\widehat{f}_{j h / k}$. Solution is approximated by

$$
\bar{f}(t)=\frac{1}{k} \sum_{i=0}^{k-1} \widehat{f}_{i h / k}(x) .
$$

\section{Error Analysis}

In this section, error analysis is studied. In the following theorems, for simplicity, we assume $T=1$ and $h=1 / \mathrm{m}$.

Theorem 9. If $\widehat{f}_{m}(x)=\sum_{i=0}^{m} f_{i} \theta_{i}(x)$ and $f_{i}=(1 /$ $\left.\Delta\left(I_{i}\right)\right) \int_{0}^{1} f(x) \theta_{i}(t) d t, i=0, \ldots, m$, then

(1) $\delta=\int_{0}^{1}\left(f(x)-\sum_{i=0}^{m} f_{i} \phi_{i}(x)\right)^{2} d x$ achieves its minimum value;

(2) $\widehat{f}_{m}(x)$ approach $f(x)$ pointwise;

(3) $\int_{0}^{1} f^{2}(x) d x=\sum_{i=0}^{\infty} f_{i}^{2}\left\|\phi_{i}\right\|^{2}$.

Proof. See [16].
Theorem 10. Assume the following.

(1) $f(x)$ is continuous and differentiable in $[-h, 1+h]$, with bounded derivative; that is, $\left|f^{\prime}(x)\right|<M$.

(2) $\widehat{f}_{i h / k}(x), i=0,1, \ldots, k-1$, are correspondingly BPFs. $h / k M B P F s, \ldots,(k-1) h / k M B P F s$ expansions of $f(x)$ base on $m+1$ EMBPFs over interval $[0,1)$.

(3) $\bar{f}(t)=(1 / k) \sum_{i=0}^{k-1} \widehat{f}_{i h / k}(x)$.

Then

$$
\left\|f(x)-\widehat{f}_{i h / k}(x)\right\|=O(h),
$$

$$
\|f(x)-\bar{f}(x)\|=O\left(\frac{h}{k}\right) \quad \text { in }[h, 1-h] .
$$

Proof. Trapezoidal rule for integral is

$$
\begin{aligned}
\int_{a}^{b} f(x) d x & =\frac{b-a}{2}(f(a)+f(b))-\frac{(b-a)^{3} f^{\prime \prime}(\eta)}{12} \\
& =\frac{b-a}{2}(f(a)+f(b))+E, \quad \eta \in[a, b],
\end{aligned}
$$

where $E$ is error of integration. Suppose $t_{i}=i / m=i$ and $I_{i}=$ $\left[t_{i-1}, t_{i}\right]$. The representation error when $f(x)$ is represented by a series of BPFs over every subinterval $\left[t_{i}, t_{i}+h / k\right], i=$ $0, \ldots, m-1$, is

$$
e_{i}(x)=f(x)-f_{i} \phi_{i}(x)=f(x)-f_{i},
$$


where

$$
f_{i}=\frac{1}{h} \int_{i h}^{(i+1) h} f(x) d x
$$

From (55),

$$
f_{i}=\frac{1}{2}\left(f\left(t_{i}\right)+f\left(t_{i}+h\right)\right)+E .
$$

It is obvious that if $f(x)=C$ (constant), then $e_{i}(x)=0$.

So, this error is computed for $f(x)=x$ in interval $\left[t_{i}, t_{i}+\right.$ $h / k], i=1, \ldots, m-1$.

For this function $E=0$, so

$$
\begin{aligned}
e_{i}(x)_{\left[t_{i}, t_{i}+h / k\right]} & =\left|x-f_{i}\right|=\left|x-\frac{t_{i}+t_{i+1}}{2}\right| \\
& =\left|x-\left(t_{i}+\frac{h}{2}\right)\right| \leq \frac{h}{2} .
\end{aligned}
$$

Then this error with BPFs is $(h / 2) M$.

Similarly, the error when $f(x)$ is represented in a series of EMBPFs over every subinterval $\left[t_{i}, t_{i}+h / k\right]$ is

$$
\begin{aligned}
e_{i}(x)_{\left[t_{i}, t_{i}+h / k\right]} & =\left|x-\left(\frac{\sum_{j=0}^{k-1}\left(t_{i}-(j h / k)+t_{i+1}-j h / k\right)}{2 k}\right)\right| \\
& =\left|x-\left(\frac{\sum_{j=0}^{k-1}\left(t_{i}-j h / k+t_{i}+h-j h / k\right)}{2 k}\right)\right| \\
& =\left|x-\left(t_{i}+\frac{h}{2}\right)-\frac{(k-1) h}{2 k}\right| \leq \frac{h}{2 k} .
\end{aligned}
$$

So, the error with EMBPFs is $(h / 2 k) M$.

For $I_{0}$ in $[0, h / k]$ we have

$$
\begin{aligned}
e_{i}(x) & =\left|x-\sum_{j=0}^{k-1} \frac{h-j h / k}{2 k}\right| \\
& =\left|x-\left(\frac{h}{2}-\frac{(k-1) h}{4 k}\right)\right|=\left|x-\left(\frac{h}{4}+\frac{h}{4 k}\right)\right| \\
& =O\left(\frac{h}{4}\right) .
\end{aligned}
$$

So, the error is $O(h / 4)$ also for $I_{n}$.
Now,

$$
\begin{aligned}
\left\|e_{i}(x)\right\|^{2} & =\int_{t_{i}}^{t_{i}+h / k}\left|e_{i}(x)\right|^{2} d x \\
& =\int_{t_{i}}^{t_{i}+h / k} \frac{h^{2}}{4 k^{2}} M^{2} d x=\frac{h^{3}}{4 k^{3}} M^{2}, \\
\|e\|^{2} & =\int_{0}^{1} e^{2}(x) d x=\int_{0}^{1}\left(\sum_{i=1}^{m} \sum_{j=0}^{k-1} e_{i}(x)\right)^{2} d x \\
& =\sum_{i=1}^{m} \sum_{j=0}^{k-1} \int_{0}^{1} e_{i}^{2}(x) d x=\sum_{i=1}^{m} \sum_{j=0}^{k-1}\left\|e_{i}(x)\right\|^{2} \\
& =\frac{1}{h} \cdot k \cdot \frac{h^{3}}{4 k^{3}} M^{2}=\frac{h^{2}}{4 k^{2}} M^{2} .
\end{aligned}
$$

We define the representation error between $f(x, y)$ and its $2 \mathrm{D}$-EMBPFs expansion, $f_{i, j}$, over every subregion $D_{i j}$, is defined as

$$
e_{i j}(x, y)=f(x, y)-f_{i j}
$$

where

$$
D_{i j}:=\left\{(x, y) \mid t_{i} \leq x \leq t_{i}+\frac{h}{k}, t_{j} \leq x \leq t_{j}+\frac{h}{k}\right\} .
$$

With Taylor's expansion and similarity to the above discussion,

$$
\|e(x, y)\|=\frac{h}{2 k} M
$$

Theorem 11. Assume that
(1) $P(w \in \Omega:\|u(\omega, t)\|<C)=1$,
(2) $\left\|k_{i}\right\|<C \quad i=1,2$.

Then

$$
\sup _{0 \leq t \leq T}\left(E(\|(u-\bar{u})\|)^{2}\right)^{1 / 2}=O\left(\frac{h}{k}\right), \quad t \in[h, 1-h] .
$$

Proof. Consider

$$
\begin{aligned}
u(t)-\bar{u}(t)= & u_{0}(t)-\overline{u_{0}}(t) \\
& +\int_{0}^{t} k_{1}(s, t) u(s)-\overline{k_{1}}(s, t) \overline{u(s)} d s \\
& +\int_{0}^{t} k_{2}(s, t) u(s)-\overline{k_{2}}(s, t) \overline{u(s)} d B(s) .
\end{aligned}
$$


TABLE 1: Mean, standard deviation, and confidence interval for error mean in Example 1 with $m=4, k=4$.

\begin{tabular}{|c|c|c|c|c|}
\hline \multirow{2}{*}{$n$} & \multirow{2}{*}{$\bar{x}_{E}$} & \multirow{2}{*}{$s_{E}$} & \multicolumn{2}{|c|}{$\% 95$ confidence interval for mean of $E$} \\
\hline & & & Lower & Upper \\
\hline 30 & $3.5678 \times 10^{-3}$ & $4.5802 \times 10^{-3}$ & $1.9287 \times 10^{-4}$ & $5.2068 \times 10^{-3}$ \\
\hline 50 & $5.0234 \times 10^{-3}$ & $7.5849 \times 10^{-3}$ & $2.9209 \times 10^{-3}$ & $7.1258 \times 10^{-3}$ \\
\hline 100 & $3.3467 \times 10^{-3}$ & $4.7983 \times 10^{-3}$ & $2.4062 \times 10^{-3}$ & $4.2871 \times 10^{-3}$ \\
\hline 125 & $4.3526 \times 10^{-3}$ & $6.3657 \times 10^{-3}$ & $3.2367 \times 10^{-3}$ & $5.4685 \times 10^{-3}$ \\
\hline
\end{tabular}

TABLE 2: Mean, standard deviation, and confidence interval for error mean in Example 1 with $m=8, k=4$.

\begin{tabular}{|c|c|c|c|c|}
\hline \multirow{2}{*}{$n$} & \multirow{2}{*}{$\bar{x}_{E}$} & \multirow{2}{*}{$s_{E}$} & \multicolumn{2}{|c|}{$\% 95$ confidence interval for mean of $E$} \\
\hline & & & Lower & Upper \\
\hline 30 & $3.0924 \times 10^{-3}$ & $5.1132 \times 10^{-3}$ & $2.6266 \times 10^{-3}$ & $4.9221 \times 10^{-3}$ \\
\hline 50 & $2.0598 \times 10^{-3}$ & $6.1477 \times 10^{-3}$ & $3.5574 \times 10^{-4}$ & $3.7635 \times 10^{-3}$ \\
\hline 100 & $1.9728 \times 10^{-3}$ & $2.2587 \times 10^{-3}$ & $1.5300 \times 10^{-3}$ & $2.4155 \times 10^{-3}$ \\
\hline 125 & $1.7054 \times 10^{-3}$ & $2.6547 \times 10^{-3}$ & $1.2400 \times 10^{-3}$ & $2.1707 \times 10^{-3}$ \\
\hline
\end{tabular}

So,

$$
\begin{aligned}
E\left(\|u-\bar{u}\|^{2}\right) \leq 3\left[E\left(\left\|\left(u_{0}-\overline{u_{0}}\right)\right\|^{2}\right)\right. & \\
+ & E\left(\left\|\int_{0}^{t}\left(k_{1} u-\overline{k_{1}} \bar{u}\right) d s\right\|^{2}\right) \\
& \left.+E\left(\left\|\int_{0}^{t}\left(k_{2} u-\overline{k_{2}} \bar{u}\right) d B(s)\right\|^{2}\right)\right] \\
\leq 3[E & \left(\left\|\left(u_{0}-\overline{u_{0}}\right)\right\|^{2}\right) \\
& +\left(\int_{0}^{t} E\left(\left\|k_{1} u-\overline{k_{1}} \bar{u}\right\|^{2}\right) d s\right) \\
& \left.+\int_{0}^{t} E\left(\left\|k_{2} u-\overline{k_{2}} \bar{u}\right\|^{2}\right) d s\right]
\end{aligned}
$$

by the Cauchy-Schwartz inequality, Itô isometry formula, and the linearity of Itô integrals in their integrands.

The first term is satisfied by last theorem:

$$
E\left(\left\|u_{0}-\overline{u_{0}}\right\|^{2}\right) \leq E\left(\frac{C^{2} h^{2}}{k^{2}}\right)=O\left(\frac{h^{2}}{k^{2}}\right) .
$$

Now,

$$
\begin{aligned}
& \|\left(k_{i}(s, t) u(t)-\overline{k_{i}}(s, t) \bar{u}(t) \|^{2}\right. \\
& \quad \leq 2\left\|\left(k_{i}-\overline{k_{i}}\right) u\right\|^{2}+2\left\|\overline{k_{i}}(\bar{u}-u)\right\|^{2} \\
& \quad \leq C \cdot\left(\left\|k_{i}-\overline{k_{i}}\right\|^{2}\right)+C \cdot\left(\|(\bar{u}-u)\|^{2}\right) .
\end{aligned}
$$

Furthermore,

$$
\left\|k_{i}-\overline{k_{i}}\right\|^{2}=O\left(\frac{h^{2}}{k^{2}}\right), \quad i=1,2 .
$$

Hence

$$
\begin{aligned}
E\left(\|u-\bar{u}\|^{2}\right) \leq 3\left[E\left(\left\|\left(u_{0}-\overline{u_{0}}\right)\right\|^{2}\right)\right. & \\
& +\int_{0}^{t} E\left(\left\|\left(k_{1} u-\overline{k_{1}} \bar{u}\right)\right\|^{2} d s\right) \\
& \left.+\int_{0}^{t} E\left(\left\|\left(k_{2} u-\overline{k_{2}} \bar{u}\right)\right\|^{2}\right) d s\right] \\
\leq & C_{0} E\left(\left\|u_{0}-\overline{u_{0}}\right\|^{2}\right)+C_{1} \int_{0}^{t} E\left(\left\|k_{1}-\overline{k_{1}}\right\|^{2}\right) d s \\
& +C_{2} \int_{0}^{t} E\left(\left\|k_{2}-\overline{k_{2}}\right\|^{2}\right) d s \\
& +C_{3} \int_{0}^{t} E\left(\|(u-\bar{u})\|^{2}\right) d s .
\end{aligned}
$$

Then by Gronwall's inequality, we get

$$
E\left(\left\|(u-\bar{u})^{2}\right\|\right) \leq C \frac{h^{2}}{k^{2}} .
$$

\section{Numerical Example}

In this section, we present an example for showing the features of the EMBPFs method in this paper. Let $X_{i}$ denote the EMBP coefficient of exact solution of the given example and 
TABLE 3: Mean, standard deviation, and confidence interval for error mean in Example 1 with $m=32$.

\begin{tabular}{|c|c|c|c|c|}
\hline \multirow{2}{*}{$n$} & \multirow{2}{*}{$\bar{x}_{E}$} & \multirow{2}{*}{$s_{E}$} & \multicolumn{2}{|c|}{$\% 95$ confidence interval for mean of $E$} \\
\hline & & & Lower & Upper \\
\hline 30 & 0.02308947 & 0.00442835 & 0.02150480 & 0.02467413 \\
\hline 50 & 0.02341165 & 0.00511389 & 0.02199415 & 0.02482915 \\
\hline 100 & 0.02364843 & 0.00524000 & 0.02262139 & 0.02467548 \\
\hline 125 & 0.02345691 & 0.00477156 & 0.02262042 & 0.02429340 \\
\hline
\end{tabular}

let $Y_{i}$ be the EMBP coefficient of computed solution by the presented method. In this example error is defined as

$$
\|E\|_{\infty}=\max _{1 \leq i \leq m}\left|X_{i}-Y_{i}\right|
$$

Example 1 (see [3]). Consider the following linear stochastic Volterra integral equation:

$$
\begin{aligned}
u(t)= & \frac{1}{12}+\int_{0}^{t} \cos (s) u(s) d s \\
& +\int_{0}^{t} \sin (s) u(s) d B(s) \quad s, t \in[0,0.5),
\end{aligned}
$$

with the exact solution $u(t)$ $(1 / 12) e^{-t / 4+\sin (t)+\sin (2 t) / 8+\int_{0}^{t} \sin (s) d B(s)}$, for $0 \leq t<0.5$.

The numerical results are shown in Tables 1 and 2 . In the tables, $n$ is the number of iterations, $\bar{x}_{E}$ is error mean, and $s_{E}$ is standard deviation of error.

Table 3 is from [3] for comparison.

In some examples by applying BPFs when $m$ increases, accuracy decreases, but in EMBPFs we achieve good accuracy by increasing $k$.

\section{Conclusion}

As some SVIEs cannot be solved analytically, in this paper we present a new technique for solving SVIEs numerically. Here, we consider a modification of the block pulse functions. Some theorems show that if EMBPFs are used for achieving numerical expansions with $k$ times more precision, there is no need to increase the number of BPFs, $k$ times, which leads to solving a system of equations with $k$ times more equations and unknowns. But the results of BPFs solution can be combined with solutions of $k-1$ systems of equations with one more unknown and nearly achieve $k$ times more precision. Parallel programming is so useful for this method. Efficiency of this method and good degree of accuracy are confirmed by a numerical example.

\section{Conflict of Interests}

The authors declare that there is no conflict of interests regarding the publication of this paper.

\section{References}

[1] J. A. D. Appley, S. Devin, and D. W. Reynolds, "Almost sure convergence of solutions of linear stochastic Volterra equations to nonequilibrium limit," Journal of Integral Equations and Applications, vol. 19, no. 4, pp. 405-437, 2007.

[2] M. A. Berger and V. J. Mizel, "Volterra equations with Itô integrals. I," Journal of Integral Equations, vol. 2, no. 3, pp. 187$245,1980$.

[3] K. Maleknejad, M. Khodabin, and M. Rostami, "Numerical solution of stochastic Volterra integral equations by a stochastic operational matrix based on block pulse functions," Mathematical and Computer Modelling, vol. 55, no. 3-4, pp. 791-800, 2012.

[4] K. Maleknejad, M. Khodabin, and M. Rostami, "A numerical method for solving $m$-dimensional stochastic Itô-Volterra integral equations by stochastic operational matrix," Computers \& Mathematics with Applications, vol. 63, no. 1, pp. 133-143, 2012.

[5] E. Pardoux and P. Protter, "Stochastic Volterra equations with anticipating coeffcients," The Annals of Probability, vol. 18, no. 4, pp. 1635-1655, 1990.

[6] Y. Shiota, "A linear stochastic integral equation containing the extended Itô integral," Mathematics Reports, Toyama University, vol. 9, pp. 43-65, 1986.

[7] C. H. Wen and T. S. Zhang, "Improved rectangular method on stochastic Volterra equations," Journal of Computational and Applied Mathematics, vol. 235, no. 8, pp. 2492-2501, 2011.

[8] D. J. Higham, X. Mao, and A. M. Stuart, "Strong convergence of Euler-type methods for nonlinear stochastic differential equations," SIAM Journal on Numerical Analysis, vol. 40, no. 3, pp. 1041-1063, 2002.

[9] M. Khodabin, K. Maleknejad, M. Rostami, and M. Nouri, "Numerical solution of stochastic differential equations by second order Runge-Kutta methods," Mathematical and Computer Modelling, vol. 53, no. 9-10, pp. 1910-1920, 2011.

[10] P. E. Kloeden and E. Platen, Numerical Solution of Stochastic Differential Equations, Applications of Mathematics, Springer, Berlin, Germany, 1992.

[11] X. Mao, Stochastic Differential Equations and Applcation, Horwood Series in Mathematics and Applications, Horwood Publishing Limited, Chichester, UK, 1997.

[12] E. Platen, "An introduction to numerical methods for stochastic differential equations," Acta Numerica, vol. 8, pp. 197-246, 1999.

[13] S. Janković and D. Ilić, "One linear analytic approximation for stochastic integrodifferential equations," Acta Mathematica Scientia, vol. 30, no. 4, pp. 1073-1085, 2010.

[14] W. J. Padgett and C. P. Tsokos, "Existence of a solution of a stochastic integral equation in turbulence theory," Journal of Mathematical Physics, vol. 12, no. 2, pp. 210-212, 1971. 
[15] R. Subramaniam, K. Balachandran, and J. K. Kim, "Existence of solutions of a stochastic integral equation with an application from the theory of epidemics," Nonlinear Functional Analysis and Applications, vol. 5, no. 1, pp. 23-29, 2000.

[16] K. Maleknejad and B. Rahimi, "Modification of block pulse functions and their application to solve numerically Volterra integral equation of the first kind," Communications in Nonlinear Science and Numerical Simulation, vol. 16, no. 6, pp. 24692477, 2011.

[17] E. Babolian, K. Maleknejad, M. Mordad, and B. Rahimi, "A numerical method for solving Fredholm-Volterra integral equations in two-dimensional spaces using block pulse functions and an operational matrix," Journal of Computational and Applied Mathematics, vol. 235, no. 14, pp. 3965-3971, 2011.

[18] K. Maleknejad, M. Shahrezaee, and H. Khatami, "Numerical solution of integral equations system of the second kind by Block-Pulse functions," Applied Mathematics and Computation, vol. 166, no. 1, pp. 15-24, 2005.

[19] B. K. Øksendal, Stochastic Differential Equations: An Introduction with Applications, Springer, New York, NY, USA, 5th edition, 1998.

[20] F. C. Klebaner, Intoduction to Stochastic Calculus with Applications, Monash University, Melbourne, Australia, 2nd edition, 2005. 


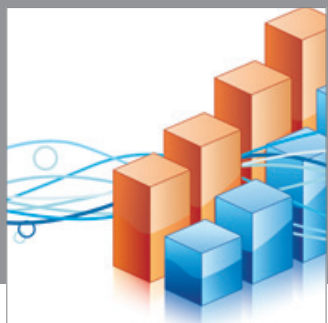

Advances in

Operations Research

mansans

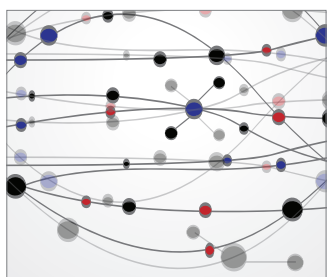

The Scientific World Journal
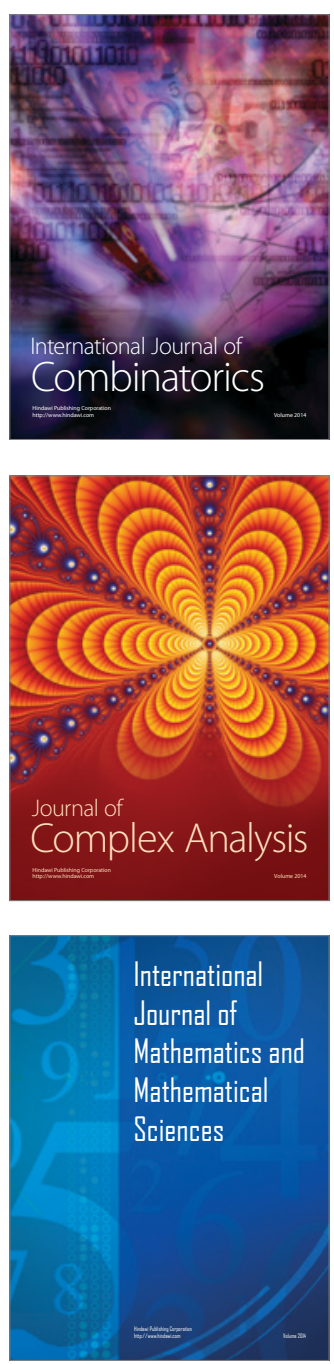
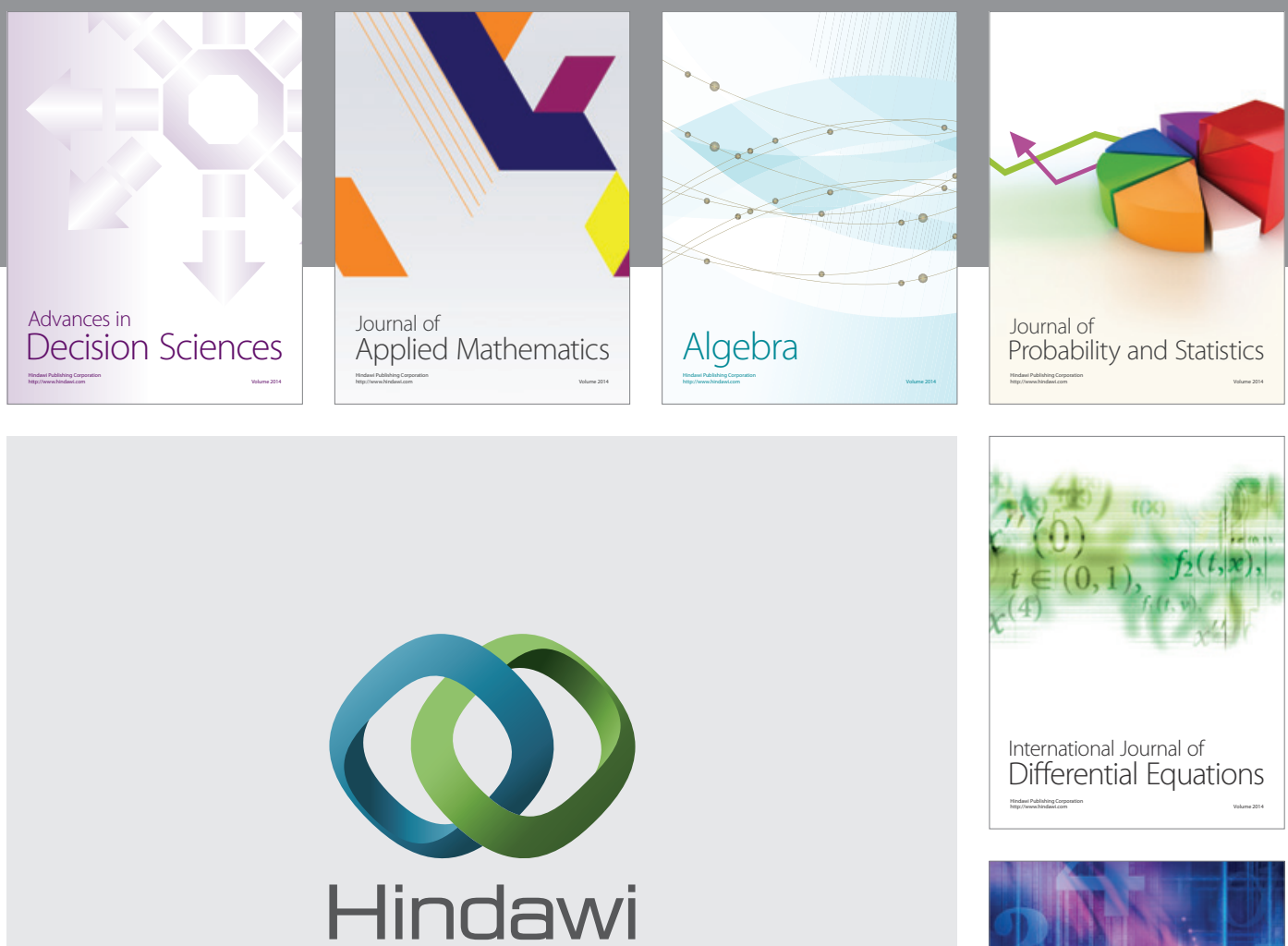

Submit your manuscripts at http://www.hindawi.com
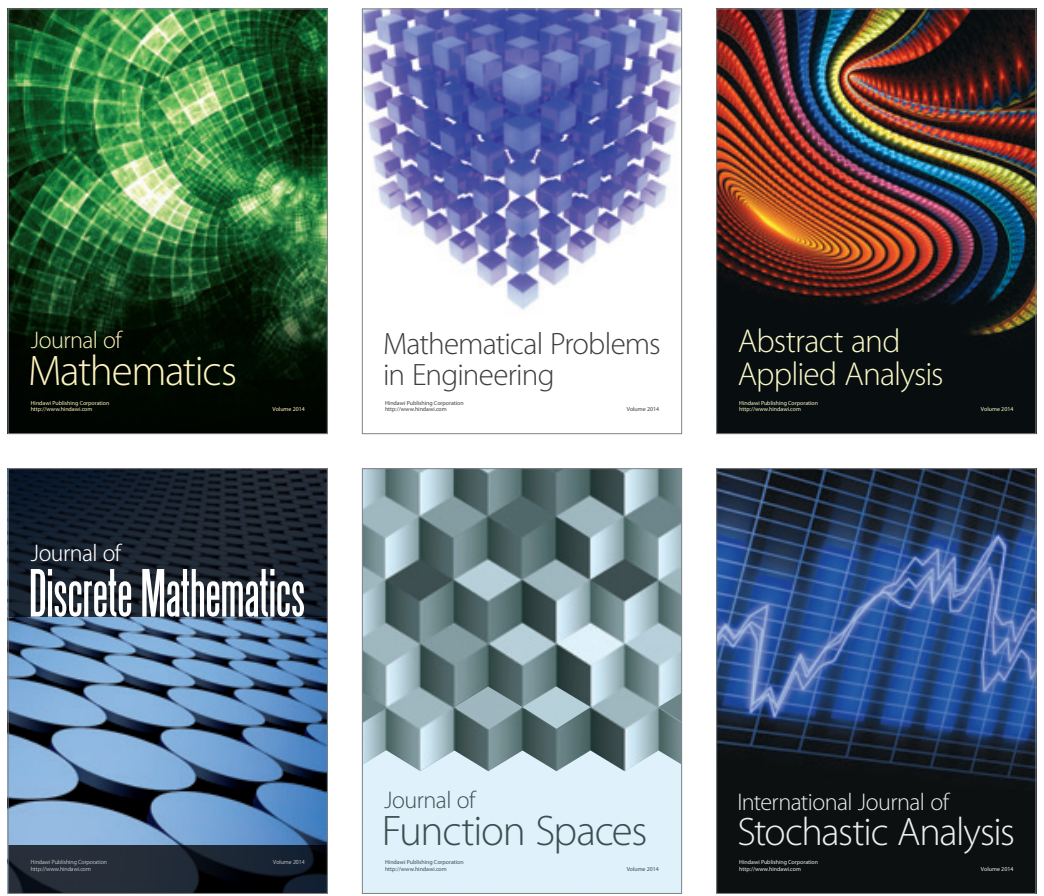

Journal of

Function Spaces

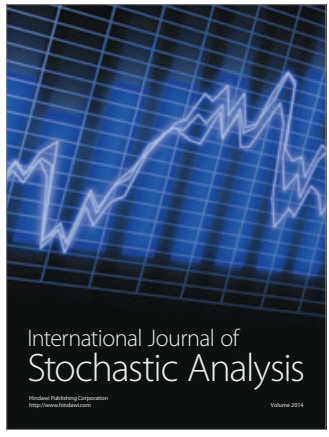

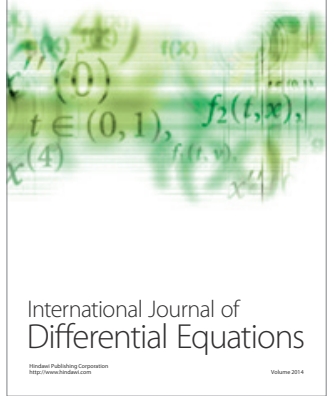
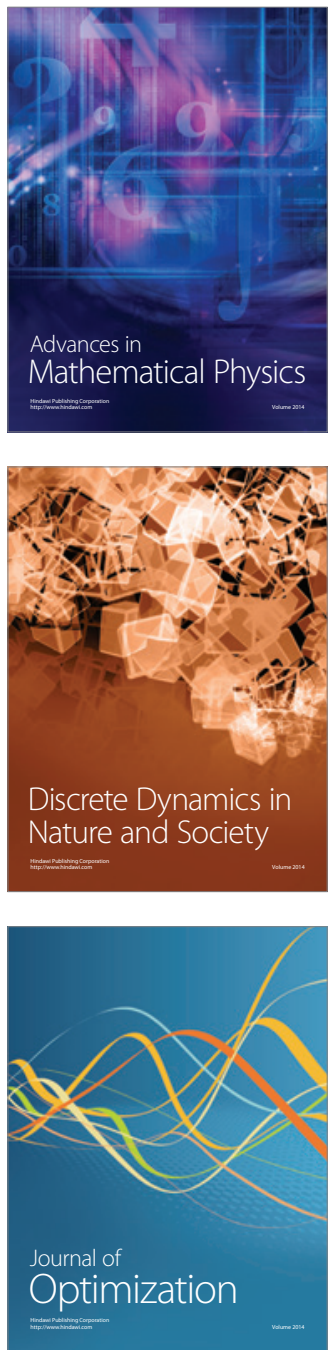